\title{
St. Gallen 2013: Brief Preliminary Summary of the Consensus Discussion
}

\author{
Nadia Harbeck $^{\mathrm{a}}$ Christoph Thomssen ${ }^{\mathrm{b}} \quad$ Michael Gnant $^{\mathrm{c}}$ \\ ${ }^{a}$ Breast Center, Department of Obstetrics and Gynecology, University of Munich, Germany \\ ${ }^{b}$ Department of Gynecology, Martin Luther University, Halle an der Saale, Germany \\ 'Department of Surgery and Comprehensive Cancer Center Vienna, Medical University of Vienna, Austria
}

\section{Keywords}

Early breast cancer - Bisphosphonates .

Endocrine therapy - Chemotherapy - Surgery .

Axillary dissection - Targeted therapy .

Neoadjuvant therapy

\section{Summary}

The 2013 St. Gallen Consensus Conference on early breast cancer provided mostly evidence-based, globally valid treatment recommendations for breast cancer care, with a broad spectrum of acceptable clinical practice. This report summarizes the results of the 2013 international panel voting procedures with regard to locoregional and endocrine treatment, chemotherapy, targeted therapy as well as adjuvant bisphosphonate use. This report is not aimed to replace the official St. Gallen Consensus publication, some recommendations may even be altered in the final paper, but should serve a preliminary rapid report of this important meeting.

\section{Introduction}

The St. Gallen Consensus Conference 2013 (March 13-16) focused again on therapy recommendations for early breast cancer which are based on evidence as well as clinical expertise of the international faculty, predominantly from Europe and the USA (table 1). The panel openly disclosed any potential conflict of interest (http://www.oncoconferences.ch); the COI committee was again chaired by Harold Burstein (Boston, USA). It was recognized as being unavoidable that individual panel members have financial relationships with commercial organizations engaged in research, innova-

\section{Schlüsselwörter}

Frühes Mammakarzinom - Bisphosphonate ·

Chemotherapie - Endokrine Therapie - Operation - Axilla . Zielgerichtete Therapie · Neoadjuvante Therapie

\section{Zusammenfassung}

Die diesjährige St. Gallen Konsensuskonferenz 2013 für das frühe Mammakarzinom gab im Wesentlichen weltweit gültige, evidenzbasierte Empfehlungen für die Behandlung, mit einem breiten Spektrum an annehmbarer klinischer Praxis. Der vorliegende Bericht fasst die Abstimmungsergebnisse des internationalen Panels im Hinblick auf Lokaltherapie, endokrine Therapie, Chemotherapie, zielgerichtete Therapie und adjuvante Bisphosphonatgabe zusammen. Dieser Bericht soll eine rasche Übersicht über die Diskussion vor Ort geben. Er kann nicht die offizielle St. Gallen Konsensus-Publikation ersetzen, einige Empfehlungen werden sicher noch überarbeitet und geändert werden.

tion, and education. None of the declared conflicts were judged to substantially impact the voting procedure and warrant exclusion of a panel member. However, members with a specific COI were asked to refrain from voting at certain questions.

The motto of this year's conference was: 'Rational Recommendations - Personalizing the Approach to Treatment of Women with Early Breast Cancer'. About 3,500 participants from 94 countries saw 2.5 days of high-level educational lectures addressing the local and systemic therapy as well as imaging issues. Next to Swiss participants, for the first time Chinese delegates constituted the largest group of attendees

\section{KARGER}

Fax +497614520714

Information@Karger.com

www.karger.com
() 2013 S. Karger GmbH, Freiburg

1661-3791/13/0082-0102\$38.00/0

Accessible online at:

www.karger.com/brc
Prof. Dr. med. Nadia Harbeck

Brustzentrum der Universität München

Standorte: Maistraße-Innenstadt und Großhadern

Marchioninistr. 15, 81377 München, Germany

Nadia.Harbeck@med.uni-muenchen.de 
Table 1. Participants of the St. Gallen 2013 international breast cancer consensus panel (as announced in the conference program - not all were present at the voting process in St. Gallen)

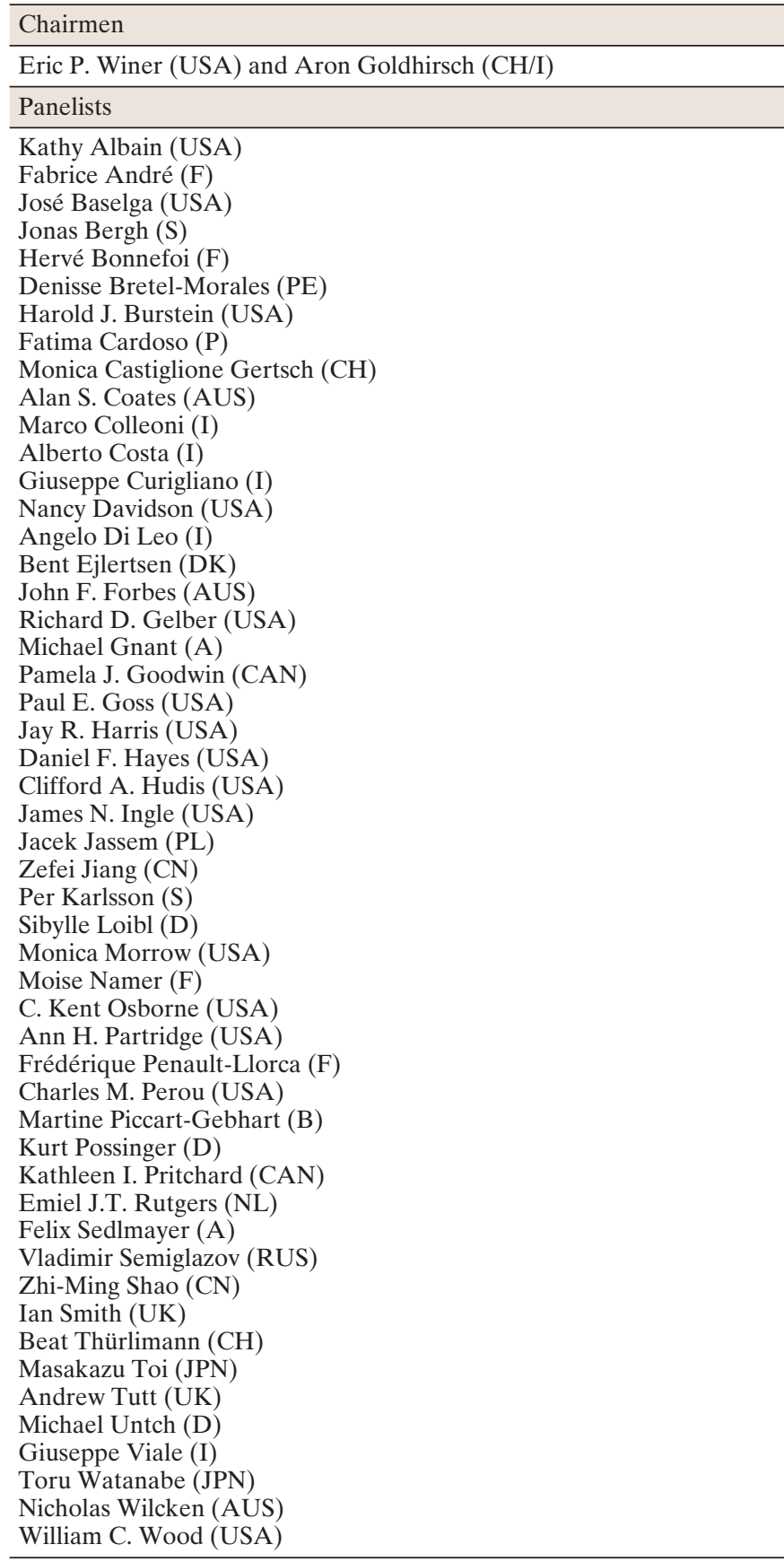

thereby demonstrating the growing international interest in this traditional meeting.

The Saturday morning consensus panel was again chaired by Aaron Goldhirsch $(\mathrm{CH} / \mathrm{I})$ and for the first time by Eric Winer (USA). Upfront, 12 areas of controversy (table 2) had been identified which deserved debate in order to agree on a range of solutions suitable for breast cancer care around the world. It was stated that clinical trials provide evidence that shows whether one treatment is better than another and help to estimate an average of outcome improvement, but not on
Table 2. Twelve areas of controversy as discussed at the panel discussion in St. Gallen, Saturday March 16, 2013

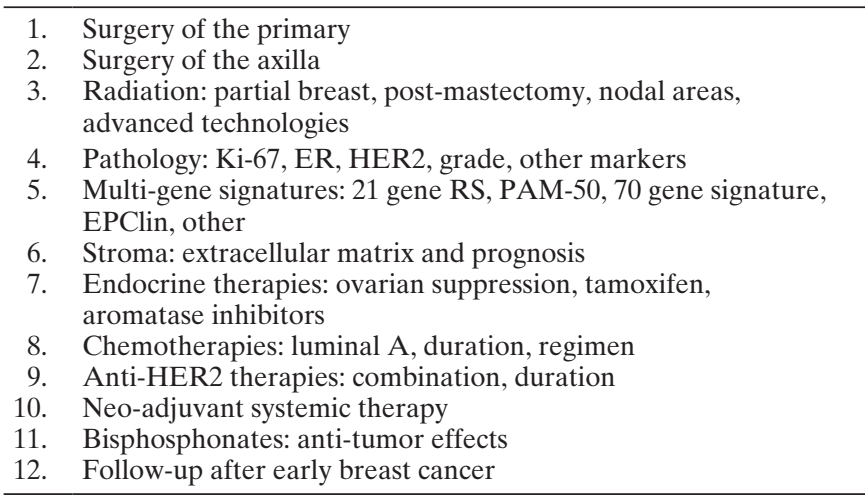

how to treat an individual patient. It was stated that treatment decisions for an individual patient therefore require interpretation and debate. The primary consideration on which the panel's voting decisions were based upon was to provide a treatment choice for women with early breast cancer by considering tumour biology, tumor extent, risks of therapy and patient preference.

In 2011, the 12th St. Gallen International Breast Cancer Conference Expert Panel had suggested for the first time to use the intrinsic biological subtypes [1] for developing therapy concepts in early breast cancer [2]: strategies for subtypes, dealing with the diversity of breast cancer. In addition to the topics of intrinsic subtypes, gene signatures and relapse risk, as well as systemic therapy covered in 2009 and 2011 [2, 3], St. Gallen 2013 also looked closely at controversial issues in loco-regional therapy, taking recent data on sentinel node excision and intraoperative radiotherapy into account.

In order to allow for an informative voting procedure, about 100 questions regarding 12 topics had been pre-prepared and agreed on by the panelists. Three possible answers were allowed: YES / NO / ABSTAIN. Abstain was to be used in case of insufficient data, no expertise on the particular issue, or conflict of interest. After each vote, the answers were summarized in percentages. Only brief panel discussions were allowed after each topic. This report summarizes the original voting questions and resulting percentages of the St. Gallen panel discussion on Saturday March 16, 2013.

\section{Surgery of the Primary Tumor}

Again, loco-regional treatment aspects were a major topic of this year's St. Gallen Consensus: Despite extensive discussions, there were no major changes in technical aspects of primary tumor resection, but it can be noted that oncoplastic techniques gain momentum in the field of breast surgery. After the pivotal publication of the ACOSOG-011 data in 2011 [4], many groups and centers have also controversially 
discussed [5] and partly or fully implemented new strategies in the surgical managements of the axilla. Hence, the operative treatment of the axilla again was another main focus in the consensus panel, both with respect to sentinel node biopsy (SNB) and axillary lymph node dissection (ALND).

Clearly, breast conserving surgery is nowadays the intended standard of care [6], and only marginal areas of controversy remain around the issue. Of note, the consensus panel of 2013 sent the strongest signal as of yet that there are virtually no absolute contraindications remaining against breast conservation. The respective votings (for absolute contraindications) were:

Young age $(<35)$ :

$90 \%$ NO $6 \%$ YES

Young age $(<40)$ :

$94 \%$ NO 2\% YES

Extensive or diffuse microcalcifications: $75 \%$ NO $19 \%$ YES

Multifocal disease:

Multicentric disease:

Tumor close to nipple:

Extensive vascular invasion:

Extensive intraductal component:

Lobular histology:

$89 \%$ NO $7 \%$ YES

$65 \%$ NO $30 \%$ YES

$96 \%$ NO $0 \%$ YES

$91 \%$ NO $6 \%$ YES

$96 \%$ NO 2\% YES

$93 \%$ NO $5 \%$ YES

With respect to relative contraindications, the consensus panel again reiterated that young age in itself is not a contraindication for breast conservation (<35 years: $61 \%$ NO, 30\% YES; $<40$ years $89 \%$ NO, 9\% YES), however recommended caution in the presence of extensive or diffuse microcalcifications (70\% YES, 15\% NO). The panel remained split about multifocality as relative contraindication for breast conservation (43\% YES, 53\% NO), but advised caution in the presence of true multicentric disease (77\% YES, $16 \% \mathrm{NO})$. Also, there was no clear opinion about a relative contraindication to breast conservation for tumors close to the nipple (43\% YES, $53 \% \mathrm{NO}$ ).

In addition, extensive vascular invasion (26\% YES, 69\% NO), extensive intraductal component (EIC; 35\% YES, 63\% $\mathrm{NO}$ ), and lobular histology were not considered even relative contraindications (5\% YES, 93\% NO) for breast conserving surgery. Furthermore, a family history of breast cancer was clearly not considered a relative contraindication for breast conserving surgery ( $4 \%$ YES, 96\% NO). The panel remained split about BRCA-1 (54\% YES, 46\% NO) and BRCA-2 (51\% YES, $47 \%$ NO) positivity as relative contraindications for breast conservation.

The panel very strongly expressed the opinion that breast conservation would not be sustainable in situations in which surgical margins remained involved despite repeated excisions (96\% YES, 2\% NO).

Despite the increasing focus on the biology of the disease, the panel did not feel that unfavorable biology, based on gene expression / sequencing, in itself constitutes a relative contraindication for breast conservation (6\% YES, 94\% NO). In the extremely rare cases of contraindications against subsequent adjuvant radiotherapy, breast conservation might not be the treatment of choice (94\% YES, $4 \%$ NO).

With respect to other surgical issues, the panel stated that nipple-sparing mastectomy without radiotherapy would be an 'acceptable' treatment (66\% YES, 21\% NO), however clearly limited this approach (elimination of radiotherapy) to those cases where the central histology margin (towards the nipple) was proven tumor-free and immediate reconstruction was performed (55\% YES, 16\% NO).

With respect to surgical planning, the panel members sent a strong signal that routine MRI (for all patients with newly diagnosed breast cancer) should not be performed (10\% YES, $89 \% \mathrm{NO}$ ), also because of the demonstrated false-positive rate of MRI with respect to multicentricity in an unselected patient population.

The never-ending issue of minimal surgical resection margins was enriched by yet another panel opinion, probably also because several globally renowned surgeons deemed 'millimeter-counting' anachronistic once again during the main sessions of the meeting: for the invasive component, the panel confirmed 'no ink on invasive tumor' as the 'appropriate minimum margin' (73\% YES, 21\% NO), however, panel members also felt that a $1 \mathrm{~mm}$ margin would be appropriate (49\% YES, $26 \%$ NO). More extensive margins were no longer considered appropriate, e.g. $3 \mathrm{~mm}$ ( $8 \%$ YES, 39\% NO). Only a minority of panel members ( $42 \%$ YES, $52 \%$ NO) thought that margins with respect to ductal carcinoma in situ should be viewed differently. A clear majority of panel members voted that minimal resection margins cannot be defined 'depending on tumor biology' (18\% YES, 78\% NO).

\section{Surgery of the Axilla}

Since the sentinel node (SN) approach is meanwhile considered a standard of care (despite many technical details without convincing evidence) for axillary staging, the 2013 consensus panel did neither discuss the principle indication nor technical aspects. However, the 'ACOSOG-Z011' question prevailed: again, and reiterating their 2011 majority, the panel declined that completion axillary dissection can be safely avoided in patients with 1-2 positive SNs and mastectomy without radiotherapy (4\% YES, 91\% NO), however was split about the issue in the presence of post-mastectomy radiotherapy (41\% YES, 43\% NO). The policy of avoiding full axillary clearance after 1-2 positive SNs was endorsed in situations of conservative surgery and radiotherapy (73\% YES, 21\% NO), including several comments that the inclusion criteria of available trial results should be considered.

Additional liberalizations for the indication for ALND were clearly declined: The panel stated strongly that ALND must be performed in cases of breast conservation who are clinically $\mathrm{N} 1$ ( $88 \%$ YES, $8 \% \mathrm{NO})$, in situations of 3 or more positive SNs (96\% YES, 5\% NO), but also recommended 
ALND in situations when information about the number of positive nodes (e.g. N4+) would be essential for the choice of chemotherapy (60\% YES, 39\% NO).

\section{Radiotherapy}

The 2013 consensus panel spent more time on modern issues of breast radiotherapy (RT) than previous panels: In general, it tried to find answers to the following questions: Is there a patient subgroup that does not need any RT? Is there a suitable subgroup for partial breast RT? Which are the risk factors requiring post-mastectomy RT to chest wall? What are indications for RT of the axilla, the supraclavicular field, the internal mammary chain? What about 15- and 16-fraction schedules for whole breast RT? Is a boost to the tumor bed really necessary in women over the age of 50 with radical microscopic resection margins?

The panel answered that there is definitely a patient subgroup who do not need RT after breast conservation $(68 \%$ YES, $20 \% \mathrm{NO}$ ), however did not vote on details regarding characterization of that group.

Limitations and or abbreviations of radiotherapy are currently a controversially discussed 'hot topic' in the field of locoregional therapy for breast cancer. Consequently, the consensus panel took votes on several related issues which could be practice-changing: 'short course radiotherapy' (40 Gy) in 15 fractions was deemed a potential new standard strategy for all patients (60\% YES, 31\% NO), and more strongly for some patients (70\% YES, 11\% NO). Short-course radiotherapy was clearly endorsed in situations where a tumor bed boost is planned (78\% YES, 4\% NO).

Partial breast irradiation after breast conserving surgery without any external beam therapy (ASTRO/ESTRO) was not endorsed in general (36\% YES, $41 \%$ NO), but was considered an option in the absence of adverse tumor pathology (49\% YES, 22\% NO). The panel did not vote on intra-operative radiotherapy.

The consensus panel strongly reiterated that postmastectomy radiotherapy (MxRT) should be considered standard in patients with $4+$ positive nodes (95\% YES, $2 \% \mathrm{NO}$ ), and recommended MxRT for patients with 1-3 positive nodes with adverse disease biology (62\% YES, 32\% NO), but not for all patients with $1-3$ positive nodes (30\% YES, 64\% NO). There was only a small majority favoring MxRT in young patients $(<40$ years of age) and $1-3$ positive nodes (55\% YES, $41 \%$ NO).

The panel clearly declined the necessity of postmastectomy radiotherapy for $\mathrm{N} 0$ patients with less than 8 nodes examined (6\% YES, 90\% NO), but recommended MxRT for cases of a positive sentinel node but no ALND performed (64\% YES, $26 \%$ NO).

With respect to young patients, the panel declined that MxRT would be necessary for all patients below the age of 40 regardless of nodal status (10\% YES, $86 \%$ NO). Also, the panel felt that MxRT should not be given to patients only on the basis of 'adverse pathology' such as grade 3 (4\% YES, 92\% NO), lymphovascular invasion (19\% YES, 77\% NO), HER2 overexpression (4\% YES, 94\% NO), or triple negative disease (2\% YES, 96\% NO). In contrast, local factors such as tumor size $>5 \mathrm{~cm}(67 \%$ YES, 29\% NO) or positive deep/ radial margins ( $82 \%$ YES, $11 \% \mathrm{NO})$, were considered indications for MxRT.

With respect to nodal areas to be included into radiation fields, the panel in general declined the notion that nodal areas should be included in irradiation fields in all patients, for the supraclavicular field ( $33 \% \mathrm{YES}, 53 \% \mathrm{NO}$ ) as well as the axilla (7\% YES, $82 \% \mathrm{NO})$ as well as the internal mammary nodes (11\% YES, 70\% NO). Because of the clear statement on not routinely including the axilla, the panel did not specifically vote on the prepared question whether the axilla should be irradiated following surgical ALND. The panel rejected the idea that the decision about including nodal areas into the radiation field should be influenced by response to neoadjuvant therapy (33\% YES, $56 \% \mathrm{NO}$ ) or the intrinsic subtype of the tumor (17\% YES, 77\% NO).

\section{Pathology}

This year, the chapter 'Prognostic and Predictive Factors' was substituted by a comprehensive 'Pathology' chapter. The focus of discussion was set on differentiation of intrinsic subtypes by means of immunohistochemistry, molecular diagnostics and multigene signatures. Also additional factors for HER2 determination, and significance of stromal factors were discussed. Attention should be paid that in many statements of this chapter the indication and type of adjuvant therapy is suggested.

\section{Subtypes}

Although prospective data are lacking, the majority felt that intrinsic subtypes may influence whether or not chemotherapy is used in the adjuvant regimen (88.9\% YES, 6.7\% NO). However, no strong agreement was achieved about the method of subtype definition. While a majority still pled against multi-gene expression array profiling being required for subtype definition (22.0\% YES, 70.0\% NO), about half of the panel opted for use of a clinic-pathologic definition (e.g., St. Gallen 2011) as sufficient for subtype definition (53.1\% YES, 38.8\% NO). Only a minority thought that the choice of cytotoxic therapy regimen should be influenced by intrinsic subtype (27.7\% YES, 68.1\% NO).

\section{Luminal A / Luminal B}

In terms of practical purposes, the majority pled for distinction between 'luminal A' and 'luminal B' (HER2-negative) tumors to be performed by estrogen receptor (ER), progesterone receptor (PR) and Ki-67 (72.9\% YES, 27.1\% NO). 
However, it was clearly stated that it can be safely determined only by laboratories participating in quality assurance programs (88.9\% YES, $8.9 \%$ NO). Of course, ER and PR alone was not considered to be sufficient for that distinction (6.1\% YES, 91.8\% NO), interestingly, also grading was not accepted as a substitute for Ki-67 (grade 3 instead of high Ki-67; 36\% YES, 64.0\% NO). Although current data show only weak concordance between immunohistochemical determination and molecular diagnostics, it was not accepted that luminal A and B subtypes can safely be determined only by molecular diagnostics (34\% YES, 60\% NO).

\section{Multigene Signatures}

A major part of the panel discussion was related to the question of routine use of multigene signatures. Interestingly, only in node-negative ER-positive HER2-negative cases, a slight majority would ask for the additional use of multigene signatures (after clinic-pathological assessment) (56.8\% YES, $43.2 \%$ NO). However, the majority of the panelists pled against application of multigene signatures in nearly all cases independently of the intrinsic subtype ( $0 \%$ YES, 97.6\% NO), they also pled against the use in nearly all ER- and/or PRpositive (HER2-negative) cases (20.8\% YES, 79.2\% NO), and against the use in node-positive ER-positive HER2-negative cases $(22.2 \%$ YES, $77.8 \%$ NO). There was an indifferent position of the panel concerning the application of multigene signatures in nearly all 'luminal B' (HER2-negative), but not 'luminal A' cases (44.4\% YES, 51.1\% NO).

The discussion was then focused on the benefit of available multigene signatures with regard to the indication of adjuvant chemotherapy in an endocrine responsive (i.e., any expression of ER and/or PR) cohort. A majority thought that the 21-gene recurrence score would predict chemotherapy response $(78 \%$ YES, 12\% NO). However, only a minority of the panelists thought that PAM50 (29.5\% YES, 40.9\% NO), a the 70 gene signature $\left(25 \%\right.$ YES, $25 \%$ NO), or the Endopredict ${ }^{\circledR}$-derived 'EPClin' algorithm (10.6\% YES, 57.4\% NO) would predict the effect of chemotherapy. Note that a substantial proportion of panelists abstained. The votes on the slightly different question whether selection of steroid hormone receptor-positive patients who might forego chemotherapy can be partially based on one of these aforementioned signatures brought similar results.

Finally, it was asked whether in some endocrine responsive cohorts (i.e., with any expression of ER and/or PR), molecular diagnostics can be omitted. The majority agreed to omit molecular diagnostics in patients with tumor size $\leq 1 \mathrm{~cm}$ (83.9\% YES, $12.9 \%$ NO) since chemotherapy would not be given anyway. On the other hand, the majority also agreed to omit molecular diagnostics in patients with inflammatory breast cancer $(93.8 \%$ YES, $4.2 \% \mathrm{NO}$ ) and in patients with 4 and more tumor infiltrated axillary lymph nodes $(91.5 \%$ YES, 6.4\% NO) since chemotherapy in these patients would be given anyway. About half of the panelists voted to omit additional molecular diagnostics for giving adjuvant chemotherapy anyway if patients had bigger tumors (e.g., $>5 \mathrm{~cm}$; $52.4 \%$ YES, $40.5 \%$ NO), or if the tumors had low expression of ER (e.g., 5\%; 55.8\% YES, 44.2\% NO). Interestingly, there was no majority to recommend adjuvant chemotherapy if tumors were undifferentiated (grade 3; 30.6\% YES, 65.3\% NO) or patients were young (e.g., < 35 years; $24.4 \%$ YES, $75.6 \%$ NO). However, it seems to be accepted worldwide that presence of 1-3 tumor infiltrated lymph nodes would not be an absolute indication for adjuvant chemotherapy $(26.3 \%$ YES, 71.1\% NO).

\section{HER2, Stroma}

With regard to the determination of HER2 status for antiHER2 treatment purposes, it was discussed whether additional information is required. However, neither heterogeneity of overexpression of HER2 (23.9\% YES, 71.7\% NO) nor polysomy 17 (10.6\% YES, 85.1\% NO) was considered mandatory additional information. Not even information on concomitant ER expression was considered to be necessary for treatment decision (40.5\% YES, 59.5\% NO). Also the degree of tumor proliferation was refused as essential information (10.4\% YES, 89.6\% NO).

As a new pathological feature of the tumor, this year the consensus panel discussed whether stromal factors should influence therapy choice in routine clinical practice. However, none of the discussed factors, neither immunocyte infiltration (11.4\% YES, 74.3\% NO), nor microvascular density $(9.5 \%$ YES, 88.1\% NO), nor stromal p16 staining (0\% YES, 97.7\% NO) was accepted.

\section{Endocrine Therapy}

\section{Establishing Standards for Premenopausal Patients}

A convincing majority of the panel members (83.3\% YES, $16.7 \%$ NO) rated tamoxifen as minimal standard ('default') in endocrine adjuvant therapy for premenopausal women. In view of the recent data of the ATLAS trials, the extension to 10 years of tamoxifen in all patients remaining premenopausal after 5 years would be considered by less than half of the panelists (42.9\% YES, 49.0\% NO), however, the majority confirmed the indication in 'some' selected patients $(88.9 \%$ YES, $8.9 \%$ NO). Interestingly, the discussion on ovarian function suppression (OFS) in addition to tamoxifen resulted not only in objection for all premenopausal patients $(14.9 \%$ YES, $80.9 \%$ NO), but also for young premenopausal patients (e.g. $<40$ years) $(40.9 \%$ YES, $50 \%$ NO).

Although some data and some guidelines [7] support the use of gonadotropin releasing hormone ( $\mathrm{GnRH})$-agonists as sole therapy in case of contraindications for tamoxifen, the option of OFS alone (without tamoxifen) was refused by the panel (24\% YES, $70 \%$ NO). Conversely, the panelists spoke for the combination of OFS with aromatase inhibition as a 
valid option in case of contraindication for tamoxifen $(85.1 \%$ YES, 8.5\% NO), but definitely not in all patients (6.3\% YES, $87.5 \%$ NO).

\section{Establishing Standards for Postmenopausal Patients}

After the passionate discussion on aromatase inhibition during the last decade, the debate is calming down to the essential questions on endocrine therapy. The decision on standards in endocrine therapy for postmenopausal patients presumably represents the general practice: some patients can be adequately treated with tamoxifen alone (63.6\% YES, $6.4 \% \mathrm{NO}$ ). If aromatase inhibition is preferred, it needs to be started upfront at least in high-risk patients $(87.2 \%$ YES, $10.6 \% \mathrm{NO}$ ), but not in all patients (47.5\% YES, $50.0 \% \mathrm{NO}$ ). Although no data were added to the results of BIG 1-98, a majority of the panelists argued for replacing upfront aromatase inhibitors by tamoxifen after 2 years $(68.1 \%$ YES, $29.8 \%$ NO).

There was a slight majority that would offer extended aromatase inhibition beyond 5 years of adjuvant endocrine treatment to patients with node-positive disease (57.8\% YES, $17.8 \% \mathrm{NO}$ ), but not to patients with node-negative disease (25.5\% YES, 66.0\% NO).

The panel differentiated the recommendation depending on the type of prior endocrine therapy. Thus, a clear majority opted for recommending extended aromatase inhibition after 5 years adjuvant tamoxifen $(83.3 \%$ YES, $11.9 \%$ NO) and also for those patients who switched from tamoxifen to an aromatase inhibitor $(73.3 \%$ YES, $11.1 \%$ NO). However, the panel majority would not recommend extension of aromatase inhibition beyond 5 years to patients who had finished prior 5 year therapy of aromatase inhibitors (35.6\% YES, $40.0 \%$ NO). Finally, it was discussed what to do in case that aromatase inhibitors would not be available or not tolerated, including patients who switched therefore to tamoxifen; although data are lacking, in those patients tamoxifen should be continued beyond 5 years of endocrine therapy (78\% YES, $8 \%$ NO).

\section{Adjuvant Chemotherapy}

The panel voted that the following factors argued for inclusion of chemotherapy into systemic therapy although concerns regarding the reproducibility of Ki-67 and grade under routine circumstances were raised:
Histological grade 3 tumor

High Ki-67

Low hormone receptor status

Positive HER2 status

Triple negative status

High 21 gene RS (e.g. > 25)

70 gene high risk

$\begin{array}{cc}\text { YES 84.4\% } & \text { NO 13.3\% } \\ \text { YES 75.5\% } & \text { NO 14.3\% } \\ \text { YES 81.6\% } & \text { NO 12.2\% } \\ \text { YES 91.8\% } & \text { NO } 8.2 \% \\ \text { YES 98.0\% } & \text { NO } 0.0 \% \\ \text { YES 93.9\% } & \text { NO } 4.1 \% \\ \text { YES 63.3\% } & \text { NO 30.6\% }\end{array}$

Any positive node

$>3$ positive nodes

Lymphovascular invasion

Young age (e.g. < 35 years)
YES $32.7 \%$ NO $67.3 \%$

YES $93.3 \%$ NO $6.1 \%$

YES $32.0 \%$ NO $64.0 \%$

YES $46.0 \%$ NO $54.0 \%$
As in 2011, the panelists voted again that luminal A tumors are less responsive to chemotherapy (83.3\% YES). They considered less intensive chemotherapy (i.e. $4 \times \mathrm{AC}, 6 \times \mathrm{CMF}$, $4 \times \mathrm{TC}$ ) adequate for luminal A tumors (61.7\% YES, $25.5 \%$ NO) and stated that in high-risk luminal A tumors based on tumor volume, chemotherapy should be added (60.0\% YES, $22.9 \%$ NO).

With respect to luminal B tumors, luminal B subtype by itself was considered to be sufficient to prescribe chemotherapy (61.2\% YES, 38.8\% NO). Ki-67 was stated to be useful in defining luminal B subtype (72.9\% YES, 20.8\% NO). In contrast to 2011, the Ki-67 cut-off of $14 \%$ was not unanimously considered as the appropriate threshold for defining luminal B subtype (23.9\% YES, 37.0\% NO). The majority felt that a cutoff between $20 \%(29.5 \%$ YES, $13.6 \% \mathrm{NO})$ and $25 \%$ (13.3\% YES, 6.7\% NO) was more appropriate. Consequently, a higher threshold was not voted on.

Several questions addressed the type of chemotherapy. For luminal B (HER2-negative) tumors, the panel preferred anthracyclines rather than CMF (70.5\% YES, 18.2\% NO). A majority voted for inclusion of taxanes (56.5\% YES, 26.1\% NO) and a chemotherapy duration of at least 6 cycles $(50.0 \%$ YES, 34.8\% NO). Dose-dense chemotherapy was not generally preferred (19.1\% YES, 68.1\% NO).

For HER2-positive disease, there was no preferred chemotherapy regimen (36.4\% YES, $61.4 \%$ NO). $68 \%$ thought that the chemotherapy for HER2-positive disease needed to contain anthracyclines (22.0\% NO, 10\% ABSTAIN) and 93.2\% thought that taxanes needed to be included (4.5\% NO).

For the basal-like or triple-negative (TNBC) phenotype, $87 \%$ of the panel thought that chemotherapy should include anthracyclines and taxanes $(6.5 \% \mathrm{NO})$. No majority was found for a regimen with more alkylating agents than for example $4 \times$ EC $(30.0 \%$ YES, $47.5 \%$ NO). Neither platinumbased regimens (14.6\% YES, 68.8\% NO) nor dose-dense chemotherapy requiring growth factors $(38.3 \%$ YES, $48.9 \%$ NO) were preferred for this subtype.

Last but not least, $72.7 \%$ of the panelists thought that there are other reasons besides tumor characteristics to prefer specific chemotherapy regimens:

Women desiring fertility

preservation

Avoiding alopecia

Comorbidities

YES $76.2 \% \quad$ NO $19.0 \%$

YES 56.5\% NO 41.3\%

YES 100\% (One of only

two unanimous votes with

$100 \%$ agreement!)

Age of the patient

Advanced age alone

YES $60.0 \%$ NO $37.1 \%$ 
Intrinsic subtype

BRCA carriers

Histological grade 3 tumor
YES $37.8 \%$

YES $20.8 \%$

YES $84.4 \%$
NO $53.3 \%$

NO $72.9 \%$

NO $13.3 \%$

\section{Anti-HER2 Therapy}

The second unanimous vote with $100 \%$ agreement was the confirmation of 1 year of trastuzumab as standard duration in early breast cancer. With regard to minimal tumor size (invasive diameter) requiring trastuzumab, $10 \%$ voted for $10 \mathrm{~mm}$, $72.5 \%$ for $5 \mathrm{~mm}$, and $17.5 \%$ for any size. $87.2 \%$ of the panelists thought that trastuzumab should be given concurrent with a taxane but only $14.3 \%$ thought that it should be given concurrently with anthracyclines ( $86.7 \% \mathrm{NO})$. If chemotherapy was contraindicated, there was a split vote regarding administration of trastuzumab with endocrine therapy alone in ER-positive disease (50\% YES, 50\% NO) but a majority of NO votes (about $85 \%$ ) in ER-negative disease. Unfortunately, during these votes the otherwise flawless voting system failed and the panel voted briefly by show of hands.

\section{Neoadjuvant Chemotherapy}

As in 2011, neoadjuvant systemic therapy was discussed separately but this year's questions focused more on concepts than substances. Only $50.9 \%$ of the panelists thought that facilitation of local therapies should be the only aim of neoadjuvant chemotherapy (NACT) $(45.3 \%$ NO). Thus, almost half of the panelists acknowledged that NACT had other aims than mere local downstaging. 95.2\% stated that after pCR to a full course of NACT, no subsequent adjuvant chemotherapy should be given. Also after failure to achieve pCR with a full course of NACT, $82.5 \%$ were opposed to subsequent adjuvant chemotherapy (10.0\% YES). After a short discussion among the panelists, the panel emphasized with a $79.2 \%$ majority that the full course of NACT should be given upfront (10.4\% NO) and with a $62.2 \%$ majority that completion by adjuvant chemotherapy should be given if pCR was reached on an incomplete course of NACT $(26.7 \% \mathrm{NO})$.

With regard to NACT in HER2-positive disease, it should contain anti-HER2 drugs (95.9\% YES). 54.3\% were against recommendation of dual HER2-targeting in the preoperative setting for HER2-positive disease (37.1\% YES).

Neoadjuvant endocrine therapy was highly accepted as a reasonable option (93.8\% YES) in postmenopausal patients with highly endocrine responsive disease (i.e., strongly positive receptors, low proliferation). $62.2 \%$ of the panelists voted for therapy duration until maximal response $(26.7 \%$ for $4-8$ months, $11.1 \%$ for $3-4$ months).

\section{Bisphosphonates}

In summary, bisphosphonates were not recommended as adjuvant therapy in addition to endocrine therapy. The first question was whether zoledronic acid, given every 6 months during adjuvant endocrine therapy, would generally be indicated to improve disease free survival; only a minority favored this option (22.5\% YES, 70\% NO). However, the voting did also not result in any subgroup recommendation, neither for premenopausal patients receiving GnRH-agonists plus tamoxifen (32.5\% YES, 58.1\% NO), nor for premenopausal patients not receiving GnRH ( 6.7\% YES, 86.7\% NO). Even for postmenopausal patients, the indication was not seen by the majority $(34.0 \% \mathrm{YES}, 61.7 \% \mathrm{NO})$. In order to be complete, the panel discussed a potential adjuvant role of denosumab; however, it was nearly consensus that zoledronic acid should not be substituted by bisphosphonates (2.2\% YES, $84.4 \%$ NO).

\section{Follow-Up after Early Breast Cancer}

For the first time, the St. Gallen consensus panel wanted to give recommendations also on aspects of surveillance after breast cancer diagnosis and treatment. Most panelists agreed that all patients should have regular follow-up visits with their surgeon/oncologist after completing their treatment (excluding those patients with long-term endocrine therapy; $70.4 \%$ YES, 25.9\% NO); however, little more than half of the panelists would also accept the option that regular follow-up supervised by a nurse specialist in person or by telephone would be acceptable for surveillance (59.1\% YES, 25.9\% NO). Finally, routine imaging as part of their follow-up apart from mammography was discussed and denied (14.9\% YES, $78.7 \%$ NO).

\section{Conclusion}

St. Gallen 2013 was an efficiently run consensus panel discussion, giving international expert opinions on 12 therapeutically relevant topics for early breast cancer. In general, the questions have evolved over the last years and managed to incorporate recent clinical discussions and controversies. The expert panel provides a corridor for internationally accepted management of early breast cancer. Evidence, clinical expertise, availability of certain drugs, diagnostic or therapeutic procedures in individual countries as well as patient preference will then determine management for the individual patient. Thus, the St. Gallen Consensus Panel recommendations may well differ from national guidelines - this was explicitly stated by Eric Winer at the beginning of the Saturday consensus. For the USA, the National Comprehensive Cancer Network (NCCN) provides widely accepted expert guidelines [8]. 
For Germany, the annually updated evidence-based Arbeitsgemeinschaft Gynäkologische Onkologie (AGO) guidelines [7] review every year peer-reviewed publications and abstracts from the major international breast cancer conferences such as ASCO (American Society of Clinical Oncology) or SABCS (San Antonio Breast Cancer Symposium) and thus provide a practical framework for evidence-based breast cancer therapy.

\section{References}

1 Perou CM, Sørlie T, Eisen MB, van de Rijn M, Jeffrey SS, Rees CA, Pollack JR, Ross DT, Johnsen H, Akslen LA, Fluge O, Pergamenschikov A, Williams C, Zhu SX, Lønning PE, Børresen-Dale AL, Brown PO, Botstein D: Molecular portraits of human breast tumours. Nature 2000;406:747-752.

$\checkmark 2$ Goldhirsch A, Wood WC, Coates AS, Gelber RD, Thürlimann B, Senn HJ; Panel members: Strategies for subtypes--dealing with the diversity of breast cancer: highlights of the St. Gallen International Expert Consensus on the Primary Therapy of Early Breast Cancer 2011. Ann Oncol 2011;22:1736-1747.
Goldhirsch A, Ingle JN, Gelber RD, et al.: Thresholds for therapies: highlights of the St. Gallen International Expert Consensus on the primary therapy of early breast cancer 2009. Ann Oncol 2009;20:1319-1329.

4 Giuliano AE, Hunt KK, Ballman KV, Beitsch PD, Whitworth PW, Blumencranz PW, Leitch AM, Saha S, McCall LM, Morrow M: Axillary dissection vs. no axillary dissection in women with invasive breast cancer and sentinel node metastasis. JAMA 2011; 305:569-575.

5 Knauer M, Gnant M, Fitzal F: Results of the first Austrian multidisciplinary expert panel on controversies in local treatment of breast cancer. Breast Care 2012;7:61-66.
6 Jakesz R, Samonigg H, Gnant M, Kubista E, Depisch D, Kolb R, Mlineritsch B, Mischinger HJ, Menzel RC, Steindorfer P, Kwasny W, Tausch C, Stierer M, Taucher S, Seifert M, Hausmaninger $\mathrm{H}$; Austrian Breast and Colorectal Cancer Study Group: Significant increase in breast conservation in 16 years of clinical trials conducted by the Austrian Breast and Colorectal Cancer Study Group. Ann Surg 2003;237:556-564.

7 AGO Kommission Mamma: Diagnosis and treatment of patients with primary and metastatic breast cancer: Guidelines of the AGO Breast Commission. www.ago-online.de.

8 NCCN National Comprehensive Cancer Network, USA. www.nccn.org. 